\title{
Suppression of $\mathrm{H}_{2}$-cooling in protogalaxies aided by trapped Ly $\alpha$ cooling radiation
}

\author{
Jemma Wolcott-Green ${ }^{1 \star(D)}$, Zoltán Haiman², and Greg L. Bryan² (0) \\ ${ }^{1}$ Department of Physics, University of California Santa Barbara, MC 9530, Santa Barbara, CA 93106, USA \\ ${ }^{2}$ Department of Astronomy, Columbia University, 550 West 120th Street, MC 5246, New York, NY 10027, USA
}

\begin{abstract}
We study the thermal evolution of UV-irradiated atomic cooling haloes using highresolution three-dimensional hydrodynamic simulations. We consider the effect of $\mathrm{H}^{-}$ photodetachment by Ly $\alpha$ cooling radiation trapped in the optically-thick cores of three such haloes, a process which has not been included in previous simulations. $\mathrm{H}^{-}$ is a precursor of molecular hydrogen, and therefore, its destruction can diminish the $\mathrm{H}_{2}$ abundance and cooling. Using a simple high-end estimate for the trapped Ly $\alpha$ energy density, we find that $\mathrm{H}^{-}$photodetachment by Ly $\alpha$ decreases the critical UV flux for suppressing $\mathrm{H}_{2}$-cooling by up to a factor of $\approx 5$. With a more conservative estimate of the Ly $\alpha$ energy density, we find the critical flux is decreased only by $\sim 15-50$ percent. Our results suggest that Ly $\alpha$ radiation may have an important effect on the thermal evolution of UV-irradiated haloes, and therefore on the potential for massive black hole formation.
\end{abstract}

Key words: cosmology: theory - early Universe - galaxies: formation - molecular processes - stars: Population III

\section{INTRODUCTION}

It has long been known that $\mathrm{H}_{2}$ is the primary coolant in primordial gas at temperatures below a few thousand Kelvin (Saslaw \& Zipov 1967). This has important implications for the first stars and protogalaxies, the reionization of the universe, and the formation of the first massive black holes (see Bromm \& Yoshida 2011, for a review). Because $\mathrm{H}_{2}$ is easily photodissociated by soft-UV photons in the Lyman-Werner (LW) bands $(11.1-13.6 \mathrm{eV})$, radiation fields from the first stars can immediately have a strong feedback effect on their environments.

Photodissociation of $\mathrm{H}_{2}$ has received particular attention in the context of gravitational collapse of haloes with virial temperatures $T_{\text {vir }} \gtrsim 10^{4} \mathrm{~K}$, in which gas is shockheated to the virial temperature and can efficiently cool via atomic line cooling, even in the presence of a strong LW radiation field. These so-called atomic cooling haloes ("ACHs") have been proposed as possible hosts of the first supermassive black hole seeds. A variety of studies have shown that the presence of a strong LW photodissociating flux can prevent $\mathrm{H}_{2}$-cooling during gravitational collapse in ACHs altogether, keeping the gas temperature near the virial temperature of the halo and thereby suppressing fragmentation on stellar-mass scales (see Inayoshi et al. 2020, for a recent

* E-mail:

jemma@astro.columbia.edu; zoltan@astro.columbia.edu; gbryan@astro.columbia.edu review). Subsequent rapid accretion rates onto a dense core $\left(\mathrm{M} \sim 0.1-1 \mathrm{M}_{\odot} \mathrm{yr}^{-1}\right)$, enabled by the elevated gas temperature, may lead to the formation of a massive $\left(10^{4-6} \mathrm{M}_{\odot}\right)$ black hole seed via a supermassive star intermediary stage (Hosokawa et al. 2012; Haemmerlé et al. 2018).

It is widely thought that in order for this so-called "direct collapse" to occur, a large critical flux $J_{\text {crit }}$ in the LW bands is required to suppress $\mathrm{H}_{2}$-cooling (but see also Inayoshi et al. 2018; Wise et al. 2019). Recent simulations have typically found $J_{\text {crit } 21}=10^{3-4}$, in the customary units $J_{\nu}=J_{21} \times 10^{-21} \mathrm{erg} \mathrm{s}^{-1} \mathrm{~cm}^{-2} \mathrm{~Hz}^{-1} \mathrm{sr}^{1}$ and normalized at the Lyman limit (Shang et al. 2010; Latif et al. 2014, 2015; Hartwig et al. 2015; Regan et al. 2014). In general, the critical flux depends sensitively on the shape of the irradiating spectrum (Sugimura et al. 2014; Agarwal et al. 2014; Wolcott-Green et al. 2017), $\mathrm{H}_{2}$ self-shielding model (Wolcott-Green et al. 2011; Hartwig et al. 2015), and rovibrational level populations (Wolcott-Green \& Haiman 2019).

The effect of "trapped" Lyman- $\alpha$ (Ly $\alpha)$ photons on fragmentation has also been considered in this context. Neutral hydrogen column densities that build up during gravitational collapse in ACHs exceed $\mathrm{N}_{\mathrm{H}} \gtrsim 10^{21} \mathrm{~cm}^{-2}$, and the resulting large optical depth in the Lyman series lines can suppress atomic cooling via Ly $\alpha$ in particular. Spaans \& Silk (2006) suggested that this could lead to a stiffer equation of state than previously assumed, thus suppressing fragmentation. However, subsequent studies have shown that atomic 
cooling via other transitions, in particular $\mathrm{H}(2 s \rightarrow 1 s)$, remain efficient even in dense cores where Ly $\alpha$-cooling is strongly suppressed (e.g. Schleicher et al. 2010).

Recently, Johnson \& Dijkstra (2017, hereafter JD17), used one-zone models to show that trapped Ly $\alpha$ photons may instead alter the thermal evolution of collapsing ACHs via photodetachment of $\mathrm{H}^{-}$, an intermediary in the primary formation reaction for $\mathrm{H}_{2}$ :

$$
\mathrm{H}^{-}+\mathrm{H} \rightarrow \mathrm{H}_{2}+\text { e. }
$$

$\mathrm{H}^{-}$can be destroyed by photons with $\mathrm{E}>0.76 \mathrm{eV}$, but previous studies have considered photodetachment only by the incident radiation field. JD17 found that while Lya photons alone do not suppress $\mathrm{H}_{2}$ abundance enough to prevent molecular cooling, when this additional photodetachment is included with an incident photodissociating flux, the critical $L W$ flux is decreased by up to a factor of $\approx 5$. Such a reduction in $J_{\text {crit }}$ would have important implications for the number density of direct collapse candidates, since the number of haloes exposed to a supercritical flux increases exponentially with decreasing $J_{\text {crit }}$ (Diikstra et al. 2008).

The goal of this study is to implement $\mathrm{H}^{-}$ photodetachment by trapped Ly $\alpha$ in a suite of threedimensional hydrodynamic simulations in order to further understand and quantify the magnitude of any reduction of $J_{\text {crit }}$ in atomic cooling haloes. We use the publicly-available ENZO code to simulate three such haloes with and without the additional $\mathrm{H}^{-}$-photodetachment by trapped Ly $\alpha$, and further compare the results using different estimates for the trapped Ly $\alpha$ energy density produced during gravitational collapse.

In one set of simulations, we adopt the same approximation for the Ly $\alpha$ energy density as in JD17 and find a similar reduction in the critical flux as found in their one-zone models: $J_{\text {crit }}(\operatorname{Ly} \alpha) / J_{\text {crit }, 0} \sim 0.2-0.8$. We also show, however, by post-processing the simulations, that their model may overestimate the amount of $\operatorname{Ly} \alpha$ produced in our haloes once the gas density reaches $\mathrm{n} \approx 10-100 \mathrm{~cm}^{-3}$, a key stage in the collapse determining whether $\mathrm{H}_{2}$-cooling is suppressed. Adopting a more conservative model for the trapped Ly $\alpha$ intensity results in a more modest reduction in the critical flux, $J_{\text {crit }}(\mathrm{Ly} \alpha) / J_{\text {crit }, 0}=0.5-1$. Our results nevertheless suggest that trapped Ly $\alpha$ may be important in the thermal evolution of UV-irradiated ACHs. A more detailed treatment of Ly $\alpha$ radiative transfer is needed to precisely determine the photodetachment rate and the resulting decrease in the critical flux.

This paper is organised as follows: We describe the numerical modeling in $\S 2$ and discuss the results in $\S 3$ We summarize our primary findings and offer conclusions in $\S 4$

\section{NUMERICAL MODELING}

\subsection{Numerical Modeling}

We use ENzd1, a publicly-available adaptive mesh refinement code, which uses an N-body adaptive particle mesh technique to follow the dark matter (DM) dynamics, and a second-order accurate piecewise parabolic method to solve

1 http://enzo-project.org

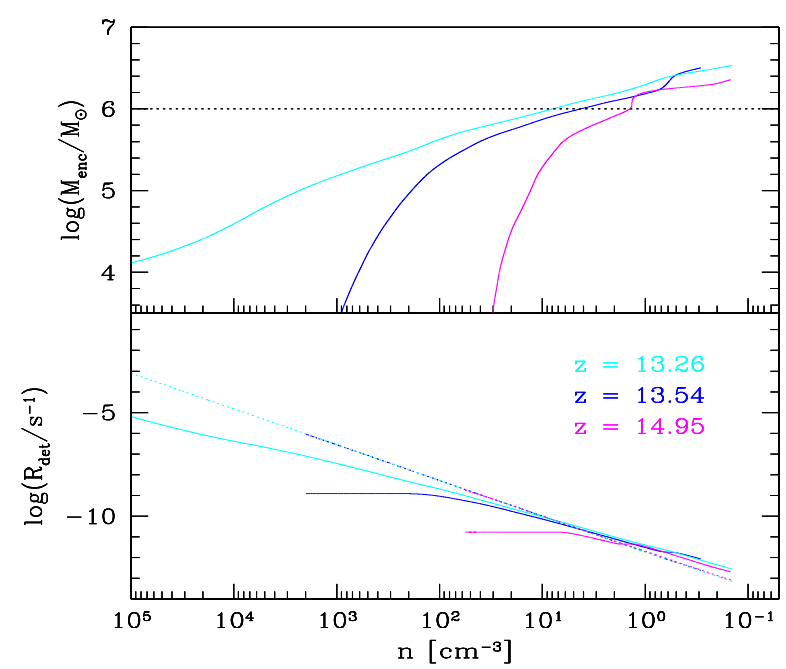

Figure 1. Top: Enclosed mass profile $M_{\mathrm{enc}}(<r, z)$ for Halo B as a function of spherically averaged density $n(r)$, shown at $z_{\text {coll }}$ (when the simulation reaches its maximum density), and two additional redshifts prior to collapse. Bottom: $\mathrm{H}^{-}$photodetachment rate due to trapped Ly $\alpha$ cooling radiation (see Equation [3) assuming a fixed mass, $M=10^{6} \mathrm{M}_{\odot}$ (dotted), or enclosed mass, $M_{\mathrm{enc}}(<r, z)$ (solid).

the hydrodynamics (see Brvan et al. 2014, for an in-depth description of the modeling). We use the 9-species nonequilibrium chemistry network in ENZO to follow the chemical evolution of gas with primordial composition. Radiative cooling by $\mathrm{H}_{2}$ is modeled with the cooling function from Galli \& Palla 1998). We also updated several of the reaction rates in the default ENZO chemistry network, as detailed in Appendix $\mathrm{A}$

Initial conditions for a simulation volume $1 h^{-1} \mathrm{Mpc}$ on a side and $128^{3}$ root grid are generated with the musid 2 package (Hahn \& Abel 2011). We initialize the simulation at $z_{\text {in }}=99$ and adopt the cosmological parameters from the Planck 2018 collaboration (Planck Collaboration et al. 2018), $\Omega_{\mathrm{m}}=0.315, \Omega_{\Lambda}=0.685 \Omega_{b}=0.0493, h=0.674$, $\sigma_{8}=0.811$, and $n=0.965$.

In order to select haloes for "zoom-in" simulations, we run an initial low-resolution DM-only simulation from $z_{\text {in }}=99$ to $z=10$, with a maximum of four levels of refinement. The ROCKSTAR halo finder (Behroozi et al. 2013) is run to find haloes with $T_{\text {vir }} \gtrsim 10^{4} \mathrm{~K}$ at $z=10$. Initial conditions are then re-generated with three nested grids enclosing the Lagrangian volume of the selected halo. With the additional nested grids, the most-refined region has an effective grid resolution of $1024^{3}$ and dark matter particle mass $\sim 100 M_{\odot}$.

High-resolution zoom simulations for three of the selected haloes are run from $z_{\text {in }}=99$ with the maximum refinement level set to 18 , resulting in a minimum cell size of $0.0298 h^{-1}$ cpc. The redshift when the simulation reaches this maximum refinement is referred to as the collapse redshift, $z_{\text {coll }}$. In order to avoid numerical effects of discrete DM

2 www-n.oca.eu/ohahn/MUSIC/ 
Table 1. Mass and virial temperature of Haloes A-C at the collapse redshift with $J=J_{\text {crit }}$ (no $\mathrm{H}^{-}$photodetachment by Ly $\alpha$.)

\begin{tabular}{llll}
\hline \hline & Halo A & Halo B & Halo C \\
\hline$z_{\text {coll }}\left(J_{\text {crit }}\right)$ & 11.87 & 13.26 & 9.36 \\
$M_{\text {tot }} / 10^{7} \mathrm{M}_{\odot}\left(z_{\text {coll }}\right)$ & 1.9 & 1.4 & 2.1 \\
$T_{\text {vir }} / 10^{3} \mathrm{~K}\left(z_{\text {coll }}\right)$ & 7.9 & 7.2 & 6.7 \\
\hline \hline
\end{tabular}

particles, the DM distribution is smoothed at a maximum refinement level of 13 . Cells are flagged for additional spatial refinement when the baryon or dark matter mass is four times greater than that of the most refined cell. In addition, the local Jeans length is always resolved by at least 16 cells in order to avoid spurious fragmentation (Truelove et al. 1997). The properties of all three haloes at their collapse redshift with $J_{21}>J_{\text {crit }}$ are shown in Table 1.

\subsection{Implementing $\mathbf{H}^{-}$photodetachment by Ly $\alpha$}

In our first set of simulations including $\mathrm{H}^{-}$photodetachment by trapped Ly $\alpha$, we utilize the model described by Johnson \& Dijkstra (2017, see their Equations 3-7), and briefly summarized here. They assume the energy radiated in Ly $\alpha$ cooling radiation balances the gravitational binding energy released by a cloud of mass $M=10^{6} \mathrm{M}_{\odot}$ collapsing on a free-fall timescale.

The derived Ly $\alpha$ energy density $u_{\alpha}$ accounts for the increased path length of a photon escaping an opticallythick medium,

$$
u_{\alpha}=M_{F} \times \frac{L_{\text {Lya }} r_{\text {cloud }}}{V_{\text {cloud }} c},
$$

where $L_{\text {Lya }}$ is the luminosity from the simple toy model above, $r_{\text {cloud }}$ is the size of the cloud, $V_{\text {cloud }}$ is the geometrical volume of the cloud, $c$ is the speed of light, and $M_{F} \sim a_{v} \tau_{\mathrm{Ly} \alpha}$, is the dimensionless path length boost. For this boost, $a_{v}$ denotes the Voigt profile, $\tau_{\mathrm{Ly} \alpha}=5.9 \times$ $10^{6}\left(\frac{N_{\mathrm{H}}}{10^{20} \mathrm{~cm}^{-2}}\right)\left(\frac{T}{10^{4} \mathrm{~K}}\right)^{-\frac{1}{2}}$ is the line-center optical depth, and $N_{\mathrm{H}}$ is the neutral hydrogen column density, found by assuming a cloud of uniform density. For an isotropic Ly $\alpha$ field within the cloud and using the cross-section for photodetachment, $\sigma_{\mathrm{H}^{-}}=5.9 \times 10^{-18} \mathrm{~cm}^{2}$ at $\mathrm{E}_{\mathrm{Ly} \alpha}=10.2 \mathrm{eV}$, they derive the photodetachment rate:

$$
\begin{aligned}
R_{\mathrm{det}} \simeq 10^{-8} \mathrm{~s}^{-1}\left(\frac{M}{10^{6} \mathrm{M}_{\odot}}\right)^{10 / 9}\left(\frac{T}{10^{4} \mathrm{~K}}\right)^{-1 / 3} & \\
\times & \left(\frac{n}{10^{2} \mathrm{~cm}^{-3}}\right)^{31 / 18}\left(\frac{B_{\alpha}}{2}\right) .
\end{aligned}
$$

Here $n$ is the density, and the parameter $B_{\alpha}$ is included to capture the possible impact of density gradients and non-uniform diffusion of spatial diffusion of $\operatorname{Ly} \alpha$ photons. These gradients and non-uniform diffusion could increase the trapped Ly $\alpha$ energy density in the center (see Appendix in JD17), but for our purposes, it is treated as a free parameter. The set of simulations we run with this rate (directly from JD17) will be referred to as "constant mass" models.

In order to evaluate the validity of this one-zone model prescription for our simulated haloes, we have examined the enclosed mass profiles at several redshift snapshots in our haloes up to the collapse redshift. These are shown for one
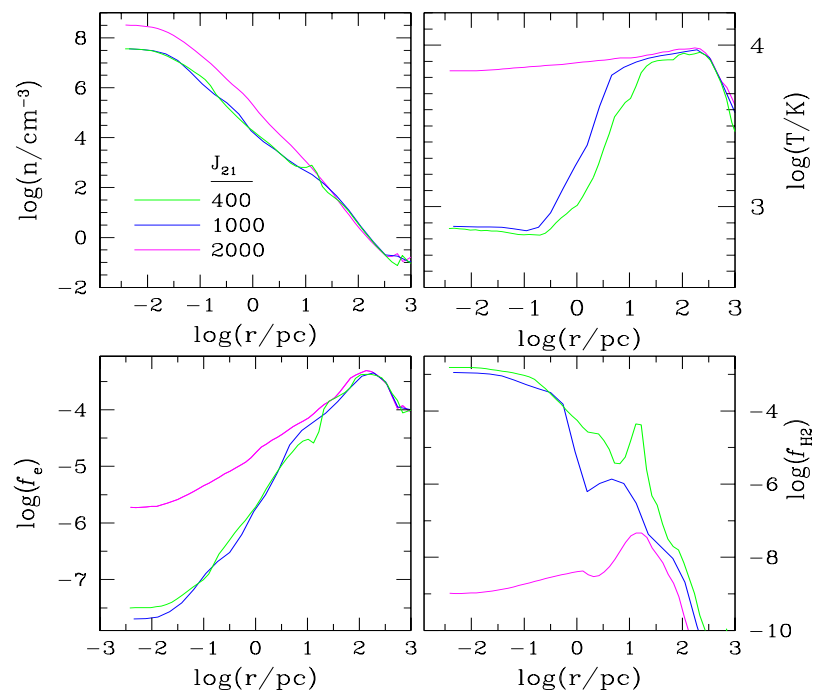

Figure 2. The profiles of spherically-averaged density (upper left), temperature (upper right), electron and $\mathrm{H}_{2}$ fractions (lower left and right, respectively) for Halo A. All profiles are at the collapse redshift of each simulation, and for varied intensity of the incident Lyman-Werner radiation: $J_{21}=400,1000,3000$.

of the haloes in the top panel of Figure 1 Because the density profiles toward the core are relatively steep, the enclosed mass $M(<r, z)$ at $n \gtrsim 10^{2} \mathrm{~cm}^{-3}$ falls rapidly below $10^{6} \mathrm{M}_{\odot}$. As a result, the photodetachment rate with fixed $\mathrm{M}=10^{6} \mathrm{M}_{\odot}$, as in JD17, is significantly larger than if the actual enclosed mass $M(<r, z)$ is instead used in Equation 3. as shown in the lower panel of Figure 1.

Using $R_{\text {det }}$ with $M(<r, z)$ self-consistently in the simulation would unfortunately require that we compute the density profile on the fly, which is beyond the scope of the current modeling. Instead, we run a set of simulations assuming $M=M\left(<r, z_{\text {coll }}\right)$, which we will refer to as the "enclosed mass" models. While this is still larger than $M(<r, z)$ at earlier redshifts, it is sufficient for the purposes of the relatively simple model used to estimate the $\operatorname{Ly} \alpha$ radiation field.

\subsection{Incident Radiation Field}

\subsubsection{Photodissociation of Molecular Hydrogen}

We adopt the commonly-used approach for modeling an incident $\mathrm{H}_{2}$-photodissociating flux with a blackbody spectrum with $\mathrm{T}_{*}=10^{5} \mathrm{~K}$ up to the Lyman limit. Ionizing photons are assumed to have been absorbed 3 , likely by neutral gas within the irradiating galaxy itself. While the Pop III IMF remains uncertain, the $\mathrm{H}_{2}$ photodissociation rate derived with this spectrum is a good approximation for metal-free

\footnotetext{
3 We do not include the characteristic saw-tooth modulation seen in the cosmological LW background spectrum as a result of absorption in the IGM (Haiman et al. 2000). The critical LW flux, $J_{\text {crit }}$, is much larger than the expected cosmological background, and is most likely to originate instead from a bright near neighbor galaxy (Visbal et al. 2014; Regan et al. 2017).
} 
Table 2. Critical fluxes in units $J_{\text {crit }, 21} / 10^{3}$ for Haloes A-C, with and without $\mathrm{H}^{-}$photodetachment by trapped Ly $\alpha$. Results are shown for constant mass, $M=10^{6} \mathrm{M}_{\odot}$, and enclosed mass, $M(<$ $\left.r, z_{\text {coll }}\right)$, models. The top row shows the value of the factor $B_{\alpha}$, which scales the trapped $\operatorname{Ly} \alpha$ photon density to allow for nonuniform density profiles and photon-diffusion (see Eq. 3).

\begin{tabular}{llllll}
\hline \hline & & \multicolumn{2}{c}{ constant mass } & \multicolumn{2}{c}{ enclosed mass } \\
\cline { 3 - 6 }$B_{\alpha}$ & 0 & 1 & 10 & 1 & 10 \\
\hline \hline Halo A & 6 & 3 & 2 & 5 & 3 \\
Halo B & 12 & 4 & 2 & 11 & 10 \\
Halo C & 7 & 6 & 2 & 7 & 4 \\
\hline \hline
\end{tabular}

starburst populations (e.g. Wolcott-Green et al. 2017). We use the fitting formula for the optically-thick $\mathrm{H}_{2}$ photodissociation rate from Wolcott-Green et al. (2011) in order to directly compare to the JD17 results; note that this fit was recently updated by Wolcott-Green \& Haiman (2019) to significantly improve the accuracy for vibrationally warm $\mathrm{H}_{2}$ $\left(\mathrm{T} \gtrsim 3000 \mathrm{~K}, n \gtrsim 10^{3} \mathrm{~cm}^{-3}\right.$ ). The self-shielding $\mathrm{H}_{2}$ column density is estimated with a local "Sobolev-like" length as the characteristic length scale:

$$
\begin{gathered}
L_{\text {char }}=\frac{\rho}{\nabla \rho}, \\
\mathrm{N}_{\mathrm{H}_{2}}=\mathrm{n}_{\mathrm{H}_{2}} \times L_{\text {char }} .
\end{gathered}
$$

This has been previously implemented in the ENZO network and Wolcott-Green et al. (2011) showed it is a more accurate local prescription than the oft-used Jeans length.

\subsubsection{Photodetachment of $\mathrm{H}^{-}$by Incident Radiation Field}

While $\mathrm{H}^{-}$photodetachment by the incident flux is not the dominant mechanism for $\mathrm{H}_{2}$-suppression with a $10^{5} \mathrm{~K}$ blackbody spectrum, it is included in our modeling with the standard rate coefficient: $k_{\mathrm{H}^{-}}=1.07 \times 10^{-11} J_{21} \mathrm{~cm}^{3} \mathrm{~s}^{-1}$.

\section{RESULTS AND DISCUSSION}

\subsection{Impact of $\mathrm{H}^{-}$Detachment by Trapped Ly $\alpha$}

In order to determine the critical flux for each of the haloes and Ly $\alpha$ models, we run the zoom simulations for each with a series of incident flux strengths. The initial runs with $J_{21}=$ $10^{3}, 5 \times 10^{3}, 10^{4}$ were analyzed at the collapse redshift to determine if $\mathrm{H}_{2}$-cooling was suppressed. Subsequently, a set of more finely-spaced flux tests (increments of $10^{3}$ in $J_{21}$ ) were run to precisely determine $J_{\text {crit }}$ required to prevent cooling below $T \approx T_{\text {vir }}$.

The resulting critical fluxes for each of our haloes and Ly $\alpha$ models are listed in Table 2 In the $B_{\alpha}=0$ cases, the only $\mathrm{H}^{-}$-photodetachment is from the incident radiation. For these, $J_{\text {crit,21 }}$ in the three haloes is found to be in the range $(6-12) \times 10^{3}$. This is a factor of $\sim 5-10$ larger than the one-zone results in JD17. Previous studies which have also found a larger critical flux in simulations as a result of hydrodynamic effects including shocks, which can increase the ionization fraction, and are not captured by the one-zone modeling (e.g. Shang et al. 2010; Latif et al. 2014, 2015).
The halo-to-halo $J_{\text {crit }}$ variation is also consistent with previous studies, which is often found to be within a factor of $\sim$ three.

We show in Figure 2 the spherically-averaged density and temperature profiles 4 at the collapse redshift for one of our simulated haloes, as well as the fractional abundances of electrons and $\mathrm{H}_{2}$. Each panel shows the results (in Halo A) for varied $J_{21}=(0.2,0.5,1.0) J_{\text {crit }}\left(B_{\alpha}=10\right)$. The results follow the typical pattern seen in previous simulations of LW-irradiated ACHs: with sub-critical flux, the $\mathrm{H}_{2}$-fraction in the dense core reaches the standard "freeze-out" value $\sim 10^{-3}$ (Oh \& Haiman 2002) resulting in robust $\mathrm{H}_{2}$-cooling and gas temperatures of a few hundred Kelvin in the inner $r \sim 0.1$ pc. Once the critical flux is reached, the $\mathrm{H}_{2}-$ fraction is suppressed, $f_{\mathrm{H}_{2}} \lesssim 10^{-7}$, and the gas temperature remains near the virial temperature of the halo $\approx 7000 \mathrm{~K}$.

\subsection{Constant Mass Models}

In the "constant mass" models, the photodetachment rate by trapped Ly $\alpha$ is identical to that implemented by JD17 (Equation 3 above, $M=10^{6} \mathrm{M}_{\odot}$ ); for direct comparison to JD17, we run two sets of models with $B_{\alpha}=1$ and 10. The critical flux in the $B_{\alpha}=1$ case is decreased by a factor of 2-3 in Haloes A and B, while in Halo $\mathrm{C}$ it is reduced by only $\sim 15$ percent compared to $B_{\alpha}=0$. The latter is similar to the $\sim 18$ per cent reduction found by JD17 in their onezone models. In our models with $B_{\alpha}=10$, the critical flux is decreased further: $J_{\text {crit }}\left(B_{\alpha}=10\right) / J_{\text {crit }, 0}=0.33,0.17,0.29$, in Haloes A, B, and C, respectively. This is as expected, since the Ly $\alpha$ detachment rate is larger, and is also consistent with the JD17 results, in which $J_{\text {crit }}\left(B_{\alpha}=10\right) \approx 0.18 J_{\text {crit }, 0}$.

Example phase diagrams of number density and temperature at $z_{\text {coll }}$ (for Halo $\mathrm{A}$ and $B_{\alpha}=1$ ) are shown in Figure 3 in the left panel the flux was sub-critical $\left(J_{21}=\right.$ $\left.2 / 3 J_{\text {crit }}\right)$ and the right panel shows results with a supercritical flux. These too are consistent with the results of previous studies; in particular, the sub-critical case shows that the shock-heated gas remains at $\sim T_{\text {vir }}$ during the collapse until the density reaches $10^{2-3} \mathrm{~cm}^{-3}$, at which point the $\mathrm{H}_{2}$ formation time becomes smaller than the dissociation time-scale, and the gas then cools (see, e.g., Shang et al. 2010, for an in-depth discussion of the relevant timescales determining $J_{\text {crit }}$ ).

\subsection{Enclosed Mass Models}

The decrease in $J_{\text {crit }}$ is smaller in our "enclosed mass" models, for which the Ly $\alpha$ energy density is calculated with $M\left(<r, z_{\text {coll }}\right)$ (derived from post-processing the haloes run with $\left.\mathrm{B}_{\alpha}=0\right)$. This is as expected, since the enclosed mass in the region where $n \gtrsim 10 \mathrm{~cm}^{-3}$ is less than $10^{6} \mathrm{M}_{\odot}$ (see Fig 1 and Table 10; therefore, this model yields a smaller Ly $\alpha$ energy density than the constant mass case at the stages of collapse that are key for determining the critical flux. Here, with $B_{\alpha}=1, J_{\text {crit }}$ is decreased in only two of the haloes (A and $\mathrm{B}$ ) and very modestly (by $\sim 10-15$ per cent). In the

\footnotetext{
${ }^{4}$ We use the publicly-available package yt (Turk et al. 2011) for simulation data analysis and visualization; see yt-project.org.
} 

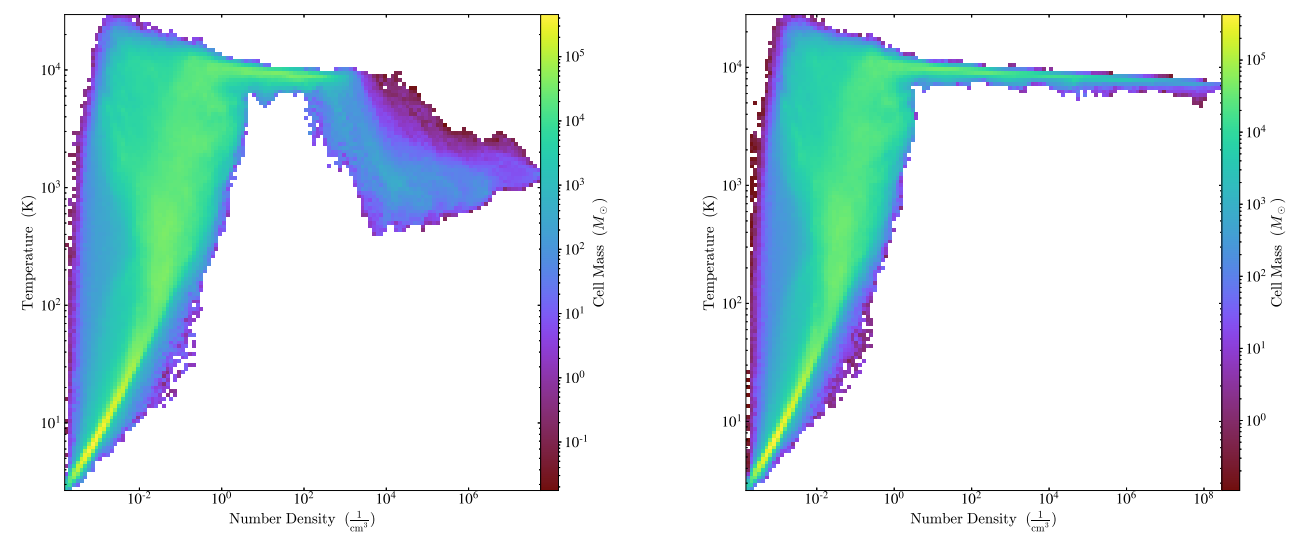

Figure 3. Phase diagrams of the sub-critical (left) and super-critical (right) runs in Halo A shown at the collapse redshift $\left(B_{\alpha}=1\right)$.

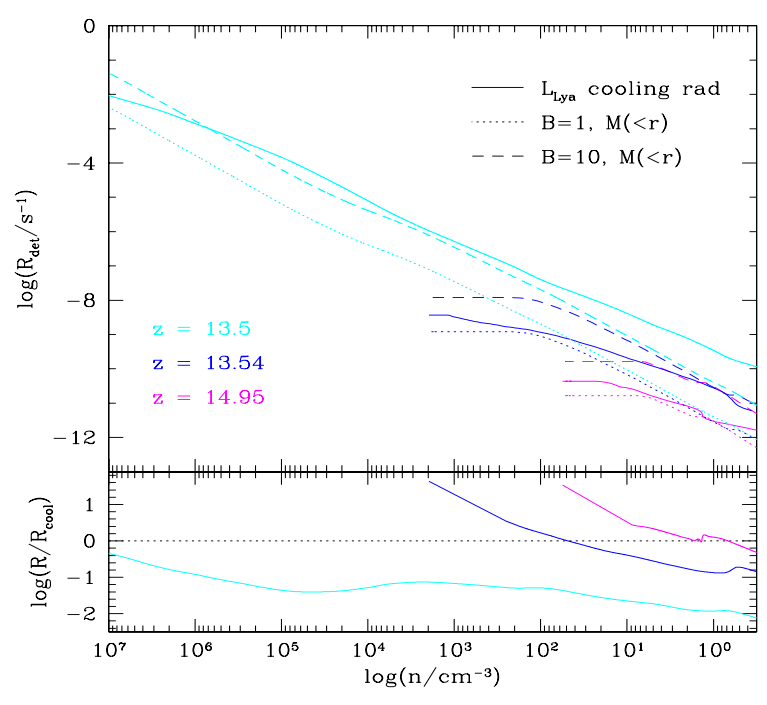

Figure 4. Top: The photodetachment rate of $\mathrm{H}^{-}$for Halo $\mathrm{B}$ at several redshifts, using Equation 3 with the enclosed mass and $B_{\alpha}=1,10$ (dotted and dashed lines, respectively). The solid line shows the same using the volume integrated cooling rate to derive a Ly $\alpha$ luminosity (rather than based on gravitational binding energy release in a free-fall time, as in JD17). Bottom: ratio of the photodetachment rate using the $B_{\alpha}=1, M\left(<r, z_{\text {coll }}\right)$ model (dotted cyan line in the upper panel) to that from the Ly $\alpha$ cooling radiation calculated at each redshift (solid lines in the upper panel). This comparison is shown because the former is used in one set of simulations (see Table 2).

$B_{\alpha}=10$ case, all three haloes see a decrease in $J_{\text {crit }}$, ranging from $\sim 15-50$ per cent (see Table 2).

Even with the modified rate using the enclosed mass, this is a somewhat crude model for estimating the Ly $\alpha$ energy density. For the sake of a "sanity check," in Figure 4 we show the photodetachment rate with Ly $\alpha$ energy density obtained directly from the volume-integrated atomic cooling rate (rather than based on the gravitational binding energy released in a free-fall time, as previously). As shown in the top panel, this " $R_{\text {cool }}$ " rate (solid lines) mostly lies between our enclosed-mass models with $B_{\alpha}=1$ and 10 (dotted and dashed lines, respectively). The exception is at the final snapshot, $z_{\text {coll }}$, when $R_{\text {cool }}$ is significantly larger than even the $B_{\alpha}=10$ model at densities below $\sim 100 \mathrm{~cm}^{-3}$.

This rough agreement with the $B_{\alpha}=1,10$ rates is reassuring that the model employed here yields a reasonable estimate for $R_{\text {det }}$; however, as discussed in $\S 2.2$, the rate implemented in our simulations actually is obtained using $M\left(<r, z_{\text {coll }}\right)$, since we do not track the enclosed mass on the fly (see $\S$ 2). Therefore, the rates implemented in our ENZO network are the $\mathrm{z}=13.5$ curves (cyan) with $B_{\alpha}=1$ and 10 (dotted and dashed).

The ratio of the $B_{\alpha}=1$ rate with $R_{\text {cool }}$ is shown in the lower panel of Figure 4 At the pre-collapse redshifts, where $n_{\max } \approx 10^{2-3} \mathrm{~cm}^{-3}$, our implemented rate with $M\left(<r, z_{\text {coll }}\right)$ diverges from $R_{\text {cool }}$ at $n \sim 10-100 \mathrm{~cm}^{-3}$ and becomes $\sim$ an order of magnitude larger at the highest densities. By the time the collapse has reached $n_{\max }=10^{7} \mathrm{~cm}^{-3}$ at $z_{\text {coll }}, R_{\text {cool }}$ is much smaller than the original rate with $B_{\alpha}=1$. This, suggests that our models may underestimate the trapped Ly $\alpha$ intensity, especially at very high densities. A more detailed study of the radiative transfer is needed in order to more precisely determine the photodetachment rates in a collapsing halo.

\subsection{Gas Inflow Rate and Mass of the Final Object}

The rate of gas inflow onto the core in ACHs is a key factor in determining the mass of the central object that can form (e.g. Inayoshi et al. 2020, and citations therein). A "critical" mass inflow rate required for SMS formation has been found to be $\sim 0.05 \mathrm{M}_{\odot} \mathrm{yr}^{-1}$ (Hosokawa et al. 2013; Schleicher et al. 2013; Haemmerlé et al. 2018).

In Figure 5 we show the mass inflow rate (upper panel) $\left(\dot{\mathrm{M}}=4 \pi \mathrm{R}^{2} \rho\left\langle v_{\mathrm{rad}}\right\rangle\right)$ for Halo $\mathrm{A}$ at the collapse redshift in the case of a supercritical (cyan) and subcritical (magenta) flux. As expected, in the supercritical case (with $T_{\text {gas }} \sim T_{\text {vir }}$ ) the mass inflow rate is significantly higher (by up to two orders of magnitude) than in the subcritical case, in which the gas has cooled via $\mathrm{H}_{2}$.

In the lower panel of Figure 5 the local accretion timescale $\left(t_{\text {acc }} \equiv \mathrm{R} /\left\langle\mathrm{v}_{\mathrm{rad}}\right\rangle\right)$ is shown for the same halo snapshot. For metal-free gas contracting on a Kelvin-Helmholtz timescale of $\sim 10^{4-5}$ years, the relevant radii, where $t_{\mathrm{acc}} \gtrsim t_{\mathrm{KH}}$, are $\sim 0.1-0.3 \mathrm{pc}$. At these radii, the mass inflow rate safely exceeds the critical rate only in the case of the supercrit- 


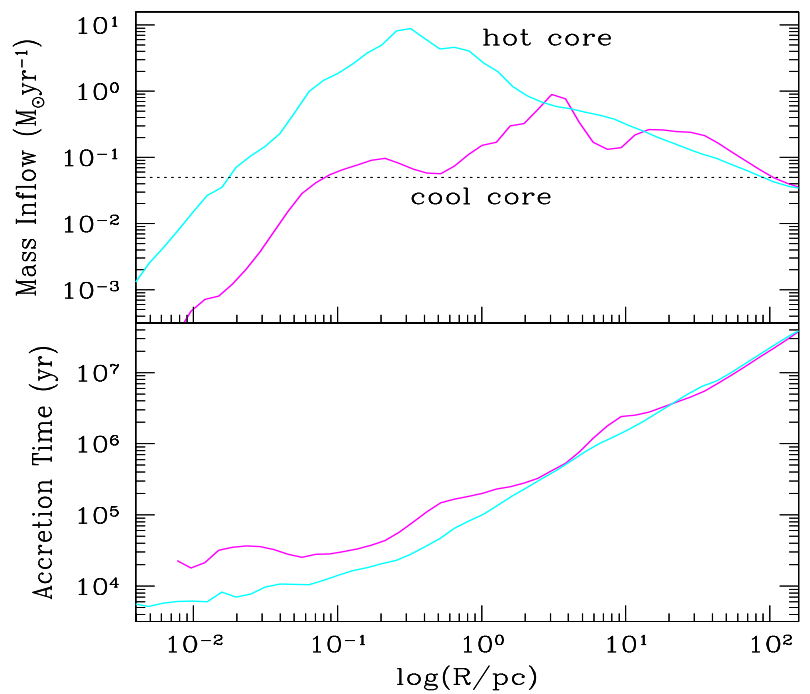

Figure 5. Lower panel: The local accretion time-scale $\equiv \mathrm{R} /\left\langle\mathrm{v}_{\mathrm{rad}}\right\rangle$ is shown for Halo A at the collapse redshift $(z=11.9)$ in the case of a supercritical flux, which prevents $\mathrm{H}_{2}$-cooling (cyan curve), and subcritical flux (magenta curve) in which the gas is able to cool via $\mathrm{H}_{2}$. Upper panel: Rate of mass inflow $\left(\dot{\mathrm{M}}=4 \pi \mathrm{R}^{2} \rho\left\langle v_{\mathrm{rad}}\right\rangle\right)$ is shown for the same halo snapshot as the lower panel. The "critical" inflow rate is marked with a dotted horizontal line. At the relevant radii for direct collapse, $\sim 0.1-0.3 \mathrm{pc}$ (see $\S 3.4$ ), the inflow safely exceeds this threshold rate only in the case of the supercritical flux (hot core). The results are qualitatively similar for the other haloes and therefore have been omitted here for clarity.

ical flux. These results are consistent with previous studies, which typically find that haloes in which $\mathrm{H}_{2}$-cooling is suppressed are more likely to maintain high accretion rates and accumulate up to $10^{4-5} \mathrm{M}_{\odot}$ of gas within the KelvinHelmholtz time.

\subsection{Depletion of $\mathbf{L y} \alpha$ by vibrationally warm $\mathrm{H}_{2}$}

There are several $\mathrm{H}_{2}$ Lyman transitions that lie close to the Ly $\alpha$ line center; therefore, in a gas with a significant $\mathrm{H}_{2}$ fraction, $\mathrm{Ly} \alpha$ can be systematically converted to $\mathrm{H}_{2}$ fluorescent emission. Neufeld (1990) showed that a large fraction of Ly $\alpha$ photons are thus converted when the $\mathrm{v}=2$, $\mathrm{J}=5,6$ states are thermally populated and the gas temperature is $\gtrsim$ a few thousand Kelvin. For example, in a cloud with $\mathrm{N}_{\mathrm{H}}=10^{20} \mathrm{~cm}^{-2}$ and $\mathrm{H}_{2}$ fraction $f_{\mathrm{H} 2}=10^{-3},>90$ per cent of Ly $\alpha$ (emitted by a central source) are converted to $\mathrm{H}_{2}$ Lyman band radiation (via the $\mathrm{B}-\mathrm{X} 1-2 \mathrm{P}[5]$ and $\mathrm{B}-\mathrm{X}$ $1-2 \mathrm{R}[6]$ transitions), before they can escape the cloud.

JD17 assume that this process is unimportant since the $\mathrm{H}_{2}$ fraction in gas exposed to a near-critical flux is small, $f_{\mathrm{H} 2} \sim 10^{-7}$. Further, they point out that even if Ly $\alpha$ photons are absorbed by vibrationally-warm $\mathrm{H}_{2}$, most of these events will result in a fluorescent radiative cascade, releasing additional photons that can photodetach $\mathrm{H}^{-}$. We note that it is also possible that this $\mathrm{Ly} \alpha$ pumping of $\mathrm{H}_{2}$ could directly contribute to the photodissociation rate and thus further suppress the $\mathrm{H}_{2}$ abundance; however, a detailed accounting of this process is beyond the scope of this work.

\section{CONCLUSIONS}

We have run a suite of high-resolution 3D hydrodynamic simulations to study the effect of trapped Ly $\alpha$ cooling radiation on the thermal evolution of UV-irradiated atomic cooling haloes. We show that the critical UV flux for suppressing $\mathrm{H}_{2}$-cooling is decreased by up to a factor of $\approx 5$ when $\mathrm{H}^{-}$photodetachment by Ly $\alpha$ is included with a simple high-end estimate of the trapped Ly $\alpha$ photon density. In models with a more conservative estimate of the trapped Ly $\alpha$ energy density, we find the critical flux is decreased by $\sim 15-50$ per cent. Our results are consistent with previous one-zone models (Johnson \& Dijkstra 2017) and suggest that Ly $\alpha$ radiation may have an important effect on the thermal evolution of UV-irradiated haloes. While we have implemented two different models for the trapped Ly $\alpha$ energy density, there remains significant uncertainty due to the difficulty of accurately computing this quantity on-thefly in simulations. This should be addressed in future work through a more detailed treatment of Ly $\alpha$ radiative transfer.

\section{ACKNOWLEDGMENTS}

This material is based upon work supported by the National Science Foundation under Award No. 1903935. JWG is grateful to Cameron Hummels for useful discussions about the simulation set-up. $\mathrm{ZH}$ and $\mathrm{GB}$ acknowledge support from NSF grant NNX15AB19G. GLB acknowledges support from NSF grants AST-1615955 and OAC-1835509.

\section{REFERENCES}

Abel T., Anninos P., Zhang Y., Norman M. L., 1997, Nature, 2, 181

Agarwal B., Dalla Vecchia C., Johnson J. L., Khochfar S., Paardekooper J.-P., 2014, MNRAS, 443, 648

Behroozi P. S., Wechsler R. H., Wu H.-Y., 2013, ApJ, 762, 109

Bromm V., Yoshida N., 2011, ARA\&A, 49, 373

Bryan G. L., Norman M. L., O'Shea B. W., Abel T., Wise J. H., Turk M. J., Reynolds D. R., Collins D. C., Wang P., Skillman S. W., 2014, ApJS, 211, 19

Dijkstra M., Haiman Z., Mesinger A., Wyithe J. S. B., 2008, MNRAS, 391, 1961

Galli D., Palla F., 1998, A\&A, 335, 403

Glover S. C. O., 2015a, MNRAS, 451, 2082

Glover S. C. O., 2015b, MNRAS, 453, 2901

Haemmerlé L., Woods T. E., Klessen R. S., Heger A., Whalen D. J., 2018, MNRAS, 474, 2757

Hahn O., Abel T., 2011, MNRAS, 415, 2101

Haiman Z., Abel T., Rees M. J., 2000, ApJ, 534, 11

Hartwig T., Glover S. C. O., Klessen R. S., Latif M. A., Volonteri M., 2015, MNRAS, 452, 1233

Hosokawa T., Omukai K., Yorke H. W., 2012, ApJ, 756, 93

Hosokawa T., Yorke H. W., Inayoshi K., Omukai K., Yoshida N., 2013, ApJ, 778, 178

Hutchins J. B., 1976, ApJ, 205, 103 
Inayoshi K., Li M., Haiman Z., 2018, MNRAS, 479, 4017

Inayoshi K., Visbal E., Haiman Z., 2020, ARA\&A, in press for vol. 58; e-print arXiv:1911.05791

Johnson J. L., Dijkstra M., 2017, A\&A, 601, A138

Kreckel H., Bruhns H., Čížek M., Glover S. C. O., Miller

K. A., Urbain X., Savin D. W., 2010, Science, 329, 69

Latif M. A., Bovino S., Grassi T., Schleicher D. R. G., Spaans M., 2015, MNRAS, 446, 3163

Latif M. A., Schleicher D. R. G., Bovino S., Grassi T., Spaans M., 2014, ApJ, 792, 78

Martin P. G., Schwarz D. H., Mandy M. E., 1996, ApJ, 461, 265

Neufeld D. A., 1990, ApJ, 350, 216

Oh S. P., Haiman Z., 2002, ApJ, 569, 558

Planck Collaboration Aghanim N., Akrami Y., Ashdown M., Aumont J., Baccigalupi C., Ballardini M., Banday A. J., Barreiro R. B., Bartolo N., 2018, A\&A, submitted, e-print arXiv:1807.06209

Regan J. A., Johansson P. H., Haehnelt M. G., 2014, MNRAS, 439, 1160

Regan J. A., Visbal E., Wise J. H., Haiman Z., Johansson P. H., Bryan G. L., 2017, Nature Astronomy, 1, 0075

Saslaw W. C., Zipoy D., 1967, Nature, 216, 976

Schleicher D. R. G., Palla F., Ferrara A., Galli D., Latif M., 2013, A\&A, 558, A59

Schleicher D. R. G., Spaans M., Glover S. C. O., 2010, ApJL, 712, L69

Shang C., Bryan G. L., Haiman Z., 2010, MNRAS, 402, 1249

Spaans M., Silk J., 2006, ApJ, 652, 902

Stancil P. C., Lepp S., Dalgarno A., 1998, ApJ, 509, 1

Sugimura K., Omukai K., Inoue A. K., 2014, MNRAS, 445, 544

Truelove J. K., Klein R. I., McKee C. F., Holliman John H. I., Howell L. H., Greenough J. A., 1997, ApJL, 489, L179

Turk M. J., Smith B. D., Oishi J. S., Skory S., Skillman S. W., Abel T., Norman M. L., 2011, ApJS, 192, 9

Visbal E., Haiman Z., Bryan G. L., 2014, MNRAS, 445, 1056

Wise J. H., Regan J. A., O'Shea B. W., Norman M. L., Downes T. P., Xu H., 2019, Nature, 566, 85

Wolcott-Green J., Haiman Z., 2019, MNRAS, 484, 2467

Wolcott-Green J., Haiman Z., Bryan G. L., 2011, MNRAS, 418, 838

Wolcott-Green J., Haiman Z., Bryan G. L., 2017, MNRAS, 469, 3329 


\section{APPENDIX A: UPDATED CHEMISTRY RATES}

Our chemistry model includes the following updates to the standard ENZO network.

Collisional dissociation of $\mathrm{H}_{2}$ by $\mathrm{H}$ :

We utilize the Martin et al. (1996) fit for collisional dissociation of $\mathrm{H}_{2}$,

$$
\mathrm{H}_{2}+\mathrm{H} \rightarrow \mathrm{H}+\mathrm{H}+\mathrm{H},
$$

including the contribution from dissociative tunneling, which has not previously been used in the ENZO network. Glover (2015a) notes this term becomes larger than direct dissociation at temperatures below $4500 \mathrm{~K}$, and found that neglecting it leads to $J_{\text {crit }}$ determinations that are erroneously large by a factor of $\sim$ two.

Associative Detachment of $\mathrm{H}^{-}$with $H$ :

We use the updated rate coefficient from Kreckel et al. (2010) for the associative detachment reaction:

$$
\mathrm{H}^{-}+\mathrm{H} \rightarrow \mathrm{H}_{2}+\mathrm{e}^{-} \text {. }
$$

In the sensitivity study by Glover (2015b), this is among the five most important reactions determining $J_{\text {crit }}$. The rate from Kreckel et al. (2010) is in good agreement with other recent determinations; however, Glover (2015b) found that the 25 per cent systematic uncertainty results in $\sim 40$ per cent uncertainty in $J_{\text {crit }}$.

Radiative recombination of $\mathrm{H}^{+}$:

We use the Case B rate from Hui+Gnedin'97 for the radiative recombination reaction,

$$
\mathrm{H}^{+}+\mathrm{e}^{-} \rightarrow \mathrm{H}+\gamma \text {. }
$$

This has been is previously included as an option in ENZO, but not always used in primordial chemistry models (e.g. Abel et al. 1997; Shang et al. 2010). In the context of an atomic cooling halo, where the mean-free path for ionizing photons is generally small, using the Case $\mathrm{B}$ rate is appropriate. Glover (2015b) note that the Case A rate is $\sim 60$ per cent larger in the relevant temperature range and therefore causes $J_{\text {crit }}$ to be $\sim 80-90$ per cent smaller in their models.

Radiative association of $H$ and $e^{-}$:

We replaced the Hutchins (1976) rate previously used in the ENZO network for radiative association reaction:

$$
\mathrm{H}+\mathrm{e}^{-} \rightarrow \mathrm{H}^{-}+\gamma .
$$

We instead use the Abel et al. (1997) rate agrees well with alternative analytic fits from Stancil et al. (1998); Galli \& Palla (1998) in the range $\mathrm{T}=10^{2}-10^{4} \mathrm{~K}$, while the Hutchins (1976) rate is significantly larger than the other three at $\mathrm{T} \geq 3000 \mathrm{~K}$. Glover $(2015 \mathrm{~b})$ find that $J_{\text {crit }}$ results using the Hutchins (1976) rate are nearly a factor of two larger than in models using any of the other three rates. Glover (2015b) also note that the Hutchins (1976) is not valid in the conditions of interest for determining $J_{\text {crit }}$ in ACHs, $\mathrm{n} \sim 10^{3} \mathrm{~cm}^{-3}, T \sim 7500 \mathrm{~K}$, and therefore recommends against using it in this context. 